\title{
Correlations and Comparison between Repeat Computed Tomography Scores, Endoscopy Scores and Symptomatic Improvement before and after Endoscopic Sinus Surgery: A Pilot Study
}

NV Deepthi, Unnikrishnan K Menon, Indudharan R Menon

\begin{abstract}
Background: Chronic rhinosinusitis (CRS) is diagnosed on the basis of symptoms, nasal endoscopic findings and computed tomography of the paranasal sinuses (CT PNS). Where indicated, functional endoscopic sinus surgery (FESS) is the surgical treatment.
\end{abstract}

Objective: To determine correlations between subjective symptom severity and objective endoscopic and radiologic findings in CRS and to compare these before and after FESS.

Materials and methods: Analysis of prospectively collected data of 20 patients undergoing FESS at a tertiary care medical center followed up for a minimum period of 6 months after surgery. RSI questionnaire-based symptom score, Lund-Mackay system-based endoscopic and CT finding scores were recorded preoperatively and at 8 weeks and 6 months postoperatively.

Results: Significant positive correlation was noted between the three parameters before surgery, especially endoscopic and radiological scores $(r=0.94$ in the latter, $p<0.01$ in all three). Postoperative improvement was statistically significant for all three parameters, even at the 6 months stage $(p<0.001)$. Postoperative correlations reduced significantly, especially between endoscopic and radiological scores.

Conclusion: This study showed the relevance of objective evaluation of routine parameters of CRS, preoperatively. It also confirmed the usefulness of FESS in indicated cases, in terms of statistical improvement of these parameters. However, repeat CT scans and the methods of scoring used by us may not be equally applicable in the long-term postoperative scenario.

Keywords: Chronic rhinosinusitis, Signs and symptoms, Nasal endoscopy, CT scan, Endoscopic sinus surgery, Statistical factor analyses.

How to cite this article: Deepthi NV, Menon UK, Menon IR. Correlations and Comparison between Repeat Computed Tomography Scores, Endoscopy Scores and Symptomatic Improvement before and after Endoscopic Sinus Surgery: A Pilot Study. Clin Rhinol An Int J 2013;6(1):32-40.

Source of support: $\mathrm{Nil}$

Conflict of interest: None declared

\section{INTRODUCTION}

Chronic rhinosinusitis (CRS) has been reported worldwide with increasing frequency in the last three decades. A s per W estern literature, it affects between 5 and $15 \%$ of studied population. ${ }^{1}$ It is often responsible for diminishing the quality of life and thereby productivity. ${ }^{2}$ Although a common medical condition, its diagnosis and prognosis depend on symptoms, findings, clinical judgement and radiological evaluation. ${ }^{3}$ This is often not very straightforward. N umerous investigators have attempted to characterize this condition based on various factors. Symptoms, computed tomography (CT) scan findings, endoscopic and surgical findings, culture and histopathology results all have featured in this regard. ${ }^{4,5}$

Once diagnosed, attempts have been made to further define the severity of CRS by using methods to assess patient symptoms via different evaluation systems. To name a few: Rhinosinusitis task force major and minor symptom criteria ${ }^{6}$ and chronic sinusitis survey (CSS), ${ }^{7} 20$ item sinonasal outcome test (SN OT 20), ${ }^{8}$ rhinosinusitis symptom inventory (RSI), ${ }^{9}$ visual analog score (VAS) questionnai re. ${ }^{10}$

$M$ anagement includes relevant investigation, followed by medical and/or surgical treatment. Computed tomography of the paranasal sinuses (CT PNS) and when indicated, functional endoscopic sinus surgeries (FESS) are now the norm. As with any aspect of medicine, there have been studies to look for correlation between the evaluation modalities and to audit the success achieved by the procedure. There are conflicting reports about the correlation between symptom, endoscopy scores and CT findings. ${ }^{11-13}$ It has been hypothesized that patients with worse disease based on CT scan experience greater symptomatic improvement after surgical treatment. There are also many reports from western literature about the symptomatic improvement and success of surgery based on endoscopy, CT and preoperative and postoperative symptom scores. ${ }^{14}$

We thought to confirm these hypotheses and findings in our surgically treated patients (FESS).

The present study aims to look at correlations among the commonly used diagnostic tools of CRS, using standardized scores, and to compare these scores before and after FESS, thus achieving a surgical audit. Specifically, we have evaluated the preoperative and postoperative symptom, endoscopic finding and CT scan scores of 20 patients with a follow-up of 6 months. Statistical correlations 
between these scores and comparisons between pre- and postoperative scores were calculated. We have been able to demonstrate significant positive correlations before surgery, significant improvement but reduced correlations after surgery.

\section{MATERIALS AND METHODS}

\section{Type of Study}

Prospective observational study.

\section{Study Setting}

Retrospective analysis of prospectively collected data of patients undergoing endoscopic sinus surgery at a tertiary care medical center catering to a population of approximately 30 million in the state of K erala in Southern India, over a 1.5 years period, for CRS with or without nasal polyposis (NP).

\section{Postgraduate Dissertation Study}

Institutional ethics committee approval was obtained.

All patients were examined, treated, operated and followed up by the same faculty of the ENT Department for the entire duration of the study.

\section{Inclusion Criteria}

1. All patients with a diagnosis of CRS with or without polyposis undergoing endoscopic sinus surgery selected from the ENT outpatient department, after getting an informed consent.

2. All patients who have received treatment with adequate antibiotics, decongestants and a 6-w eek course of topical steroids, systemic antihistamines and systemic steroids.

3. Preoperative CT scans would be taken after the aforementioned medical treatment.

4. All patients who comply with regular postoperative follow-up and management protocol, including nasal douching, resumption of antiallergic treatment and willingness for postoperative CT after 8 weeks and 6 months of surgery.

\section{Exclusion Criteria}

1. Those with other morbidities like diabetes mellitus, malignancy, immunocompromised status; also pregnant women and breastfeeding mothers.

2. Those with a history of nasal and paranasal sinus surgery in the past.

3. Those with histological or radiological documentation of invasive fungal sinusitis.
4. Those who are not compliant with the prescribed medical and surgical treatment and follow-up.

Twenty consecutive patients diagnosed with CRS and refractory to medical therapy (topical nasal steroid spray for 6 weeks, systemic steroids for 15 days, antibiotics for 7 days and antihistamines for 3 weeks) were chosen. U pon selection, consent was taken from the patient or his/her relative and they underwent preoperative work up. Diagnostic nasal endoscopy using rigid $0^{\circ}$ scope was done both at first visit and at the end of 6 weeks. On the same day, they were subjected to CT PNS, followed by FESS within a weeks' time.

Our preferred modality was multislice CT with a $3 \mathrm{~mm}$ slice thickness at $2000 \mathrm{H}$ ounsfield unit (125 kV and 450 $\mathrm{mA}$ ), without contrast media, in axial and coronal plains, and with images viewable under soft tissue and bone window settings. The reading and L und-M ackay based scoring of these scans were done by the same senior ENT doctor in our department.

Standard steps of FESS using $0^{\circ}, 30^{\circ}$ and $70^{\circ}$ telescopes were performed. Complete clearance of the disease was achieved for permanent drainage and ventilation of the affected sinuses taking care to preserve the underlying mucosa. After surgery, all patients were reviewed every week in the first postoperative month and every month after that for 6 months. A II patients received steroid nasal sprays and antihistamines in the postoperative period. Their symptom scores, endoscopic scores and radiological scores were recorded preoperatively (i.e. after 6 w eeks of medical treatment), and at 8 weeks and 6 months postoperatively. Total duration of the study was approximately 18 months; this being the time required for selecting and conducting it on 20 patients.

\section{Scoring Systems}

- Symptom score (SS) - Questionnaire based on the RSI

- Nasal endoscopic score (ES) - L und-M ackay

- CT PNS score (LM) - L und-M ackay

These are detailed below.

\section{Modified RSI Scoring}

Instruction to patient as regards the scoring pattern: 'Please rate the following individual item based on your average symptoms after medical treatment and postoperatively in the respective columns. Please circle the appropriate number.'

This score sheet was given at 8 weeks after surgery and again at 6 months. 


\begin{tabular}{|c|c|c|c|c|c|c|c|}
\hline Symptoms & & Absent & Very mild & Mild & Moderate & Severe & Very severe \\
\hline 1. Facial pain/pressure & Preoperative & 0 & 1 & 2 & 3 & 4 & 5 \\
\hline & Postoperative & 0 & 1 & 2 & 3 & 4 & 5 \\
\hline 2. Facial congestion & Preoperative & 0 & 1 & 2 & 3 & 4 & 5 \\
\hline & Postoperative & 0 & 1 & 2 & 3 & 4 & 5 \\
\hline 3. Nasal obstruction & Preoperative & 0 & 1 & 2 & 3 & 4 & 5 \\
\hline & Postoperative & 0 & 1 & 2 & 3 & 4 & 5 \\
\hline 4. Purulent nasal discharge/ & Preoperative & 0 & 1 & 2 & 3 & 4 & 5 \\
\hline postnasal drip & Postoperative & 0 & 1 & 2 & 3 & 4 & 5 \\
\hline \multirow[t]{2}{*}{ 5. Reduced sense of smell } & Preoperative & 0 & 1 & 2 & 3 & 4 & 5 \\
\hline & Postoperative & 0 & 1 & 2 & 3 & 4 & 5 \\
\hline \multirow[t]{2}{*}{ 6. Headache } & Preoperative & 0 & 1 & 2 & 3 & 4 & 5 \\
\hline & Postoperative & 0 & 1 & 2 & 3 & 4 & 5 \\
\hline \multirow[t]{2}{*}{ 7. Fever } & Preoperative & 0 & 1 & 2 & 3 & 4 & 5 \\
\hline & Postoperative & 0 & 1 & 2 & 3 & 4 & 5 \\
\hline \multirow[t]{2}{*}{ 8. Bad breath (halitosis) } & Preoperative & 0 & 1 & 2 & 3 & 4 & 5 \\
\hline & Postoperative & 0 & 1 & 2 & 3 & 4 & 5 \\
\hline \multirow[t]{2}{*}{ 9. Fatigue/tiredness } & Preoperative & 0 & 1 & 2 & 3 & 4 & 5 \\
\hline & Postoperative & 0 & 1 & 2 & 3 & 4 & 5 \\
\hline \multirow[t]{2}{*}{ 10. Dental pain } & Preoperative & 0 & 1 & 2 & 3 & 4 & 5 \\
\hline & Postoperative & 0 & 1 & 2 & 3 & 4 & 5 \\
\hline \multirow[t]{2}{*}{ 11. Cough } & Preoperative & 0 & 1 & 2 & 3 & 4 & 5 \\
\hline & Postoperative & 0 & 1 & 2 & 3 & 4 & 5 \\
\hline \multirow[t]{2}{*}{ 12. Ear pain/fullness } & Preoperative & 0 & 1 & 2 & 3 & 4 & 5 \\
\hline & Postoperative & 0 & 1 & 2 & 3 & 4 & 5 \\
\hline
\end{tabular}

\section{Lund-Mackay Endoscopy Staging System}

Preoperative endoscopic appearance

\begin{tabular}{lll}
\hline Characteristic & Right & Left \\
\hline Polyps & $0,1,2,3$ & $0,1,2,3$ \\
Edema & $0,1,2$ & $0,1,2$ \\
Discharge & $0,1,2$ & $0,1,2$ \\
\hline
\end{tabular}

Polyps: 0 -absence of polyps; 1 - polyps in middle meatus only; 2 - polyps beyond the middle meatus but notcompletely obstructing the nose; 3 -polyps completely obstructing the nose; Edema: $0-$ absent; 1 -mild; 2 -severe; Discharge: 0 -no discharge; 1 -clear thin discharge; 2-thick purulent discharge.

\section{Postoperative}

\begin{tabular}{lll}
\hline Characteristic & Right & Left \\
\hline Scarring & $0,1,2$ & $0,1,2$ \\
Crusting & $0,1,2$ & $0,1,2$ \\
\hline
\end{tabular}

Scarring: 0-absent; 1-mild; 2 - severe

Crusting: 0 -absent; 1 -mild; 2 -severe

\section{Lund-Mackay Radiologic grading of CT PNS}

\begin{tabular}{lll}
\hline Sinus system & Right & Left \\
\hline Maxillary & $0,1,2$ & $0,1,2$ \\
Anterior ethmoidal & $0,1,2$ & $0,1,2$ \\
Posterior ethmoidal & $0,1,2$ & $0,1,2$ \\
Sphenoidal & $0,1,2$ & $0,1,2$ \\
Frontal & $0,1,2$ & $0,1,2$ \\
Ostiomeatal complex (OMC) & 0,2 & 0,2 \\
\hline Total & $0-12$ & $0-12$ \\
\hline
\end{tabular}

All sinus systems, except OMC: 0 -clear; 1 - partial opacification; 2-total opacification; Ostiomeatal complex: 0- not occluded; 2-occluded

\section{STATISTICAL ANALYSIS}

Statistical analysis was done using SPSS (version 11) software. Categorical variables were presented in terms of numbers and percentages. Numerical variables were presented in terms of mean and standard deviation. Probability value less than 0.05 was considered to be statistically significant. The nonparametric W ilcoxon signed rank statistical analysis test was used for testing the mean effect of improvement after surgery. Pearson correlation coefficient ( $r$ ) was used to find out the correlation between two variables.

\section{RESULTS}

During the study period, all patients who required FESS were evaluated for eligibility for inclusion. Of these, 20 were selected as per our inclusion and exclusion criteria.

M ean age: $40.9 \pm 15.34$

Sex distribution: 14 males, 6 females

\section{Comparisons}

\section{Symptom Score (SS)}

- Range: 0 to 5

- M ean preoperative value: $2.103 \pm 0.383$

- Mean 8-week postoperative value: $0.423 \pm 0.340$ $(p<0.001)$ (Fig. 1 and Table 1)

- Mean 6-month postoperative value: $0.69 \pm 0.50$ $(p<0.001)$ (Table 2) 
Correlations and Comparison between Repeat Computed Tomography Scores, Endoscopy Scores and Symptomatic

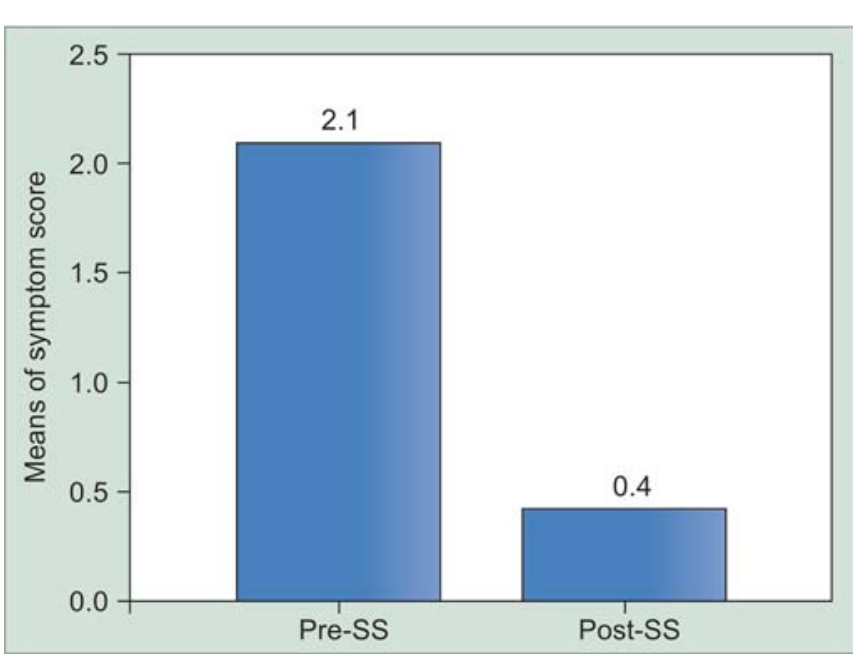

Fig. 1: The mean of symptom scores, pre- and 8-week postoperatively, using the modified RSI questionnaire (Pre-SS: Preoperative symptom score; Post-SS: Postoperative symptom score)

\begin{tabular}{|c|c|c|c|}
\hline Variable & Mean & Standard deviation & $\mathrm{p}$-value \\
\hline Pre-SS & 2.10 & 0.38 & 0.001 \\
\hline Post-SS & 0.42 & 0.34 & 0.001 \\
\hline Pre-LM & 15.05 & 5.73 & 0.001 \\
\hline Post-LM & 2.45 & 2.56 & 0.001 \\
\hline Pre-ES & 7.40 & 2.89 & 0.001 \\
\hline Post-ES & 2.10 & 1.45 & 0.001 \\
\hline
\end{tabular}

Pre-SS: Preoperative symptom score;

Post-SS: Postoperative symptom score;

Pre-LM: Preoperative radiological (Lund-Mackay) score;

Post-LM: Postoperative radiological (Lund-Mackay) score;

Pre-ES: Preoperative endoscopic score;

Post-ES: Postoperative endoscopic score

Table 2: Comparison of the three parameters of CRS
$\begin{aligned} & \text { before and at } 6 \text { months after FESS } \\
& \text { Variable }\end{aligned}$
\begin{tabular}{lrcc} 
Mean & Standard deviation & p-value \\
\hline Pre-SS & 2.10 & 0.38 & 0.001 \\
Post-SS6 & 0.69 & 0.50 & 0.001 \\
Pre-LM & 15.05 & 5.73 & 0.001 \\
Post-LM6 & 4.85 & 4.64 & 0.001 \\
Pre-ES & 7.40 & 2.89 & 0.001 \\
Post-ES6 & 2.85 & 1.09 & 0.001 \\
\hline
\end{tabular}

Pre-SS: Preoperative symptom score;

Post-SS 6: Postoperative symptom score after 6 months;

Pre-LM: Preoperative radiological (Lund-Mackay) score;

Post-LM6: Postoperative radiological (Lund-Mackay) score after 6 months;

Pre-ES: Preoperative endoscopic score;

Post-ES6: Postoperative endoscopic score after 6 months

Thus, there was significant statistical improvement in the symptoms after the surgery, which was maintained till 6 months.

\section{Endoscopic Score (ES)}

- M ean preoperative value: $7.40 \pm 2.89$

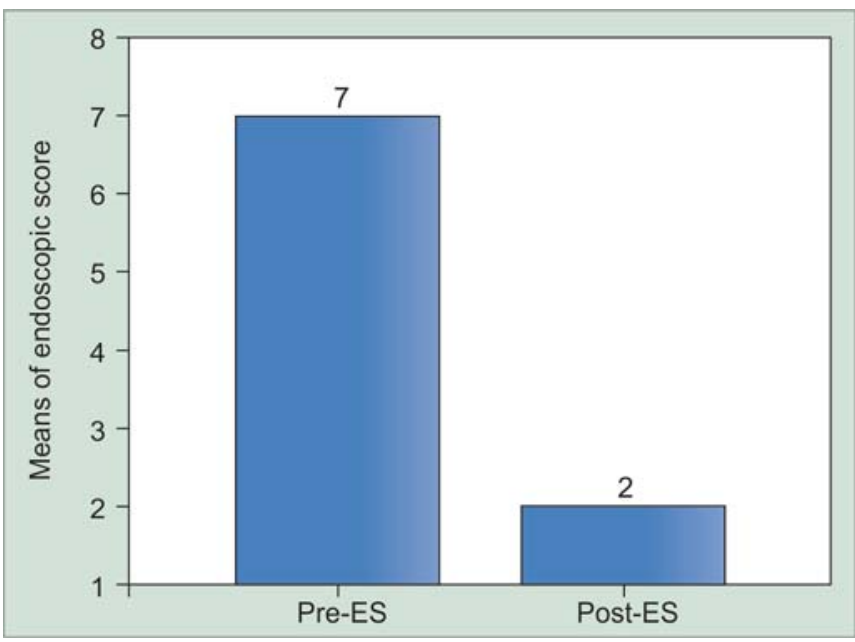

Fig. 2: The mean of endoscopy scores, pre- and 8-week postoperatively, using Lund-Mackay (endoscopic) scoring system (PreES: Preoperative endoscopic score; Post-ES: Postoperative endoscopic score)

- Mean 8-week postoperative value: $2.10 \pm 1.45$ $(p<0.001)$ (Fig. 2 and Table 1$)$

- Mean 6-month postoperative value: $2.85 \pm 1.09$ $(p<0.001)$ (Table 2)

Significant improvement was demonstrated in the endoscopic findings after surgery.

\section{Radiological Score (LM)}

- Mean preoperative value: $15.05 \pm 5.73$

- Mean 8-week postoperative value: $2.45 \pm 2.56$ $(p<0.001)$ (Fig. 3 and Table 1$)$

- Mean 6-month postoperative value: $4.85 \pm 4.64$ $(p<0.001)$ (Table 2)

Obvious improvement was seen in the CT PNS images. The variation in the mean and standard deviation is probably due to the difference in the preoperative and postoperative values.

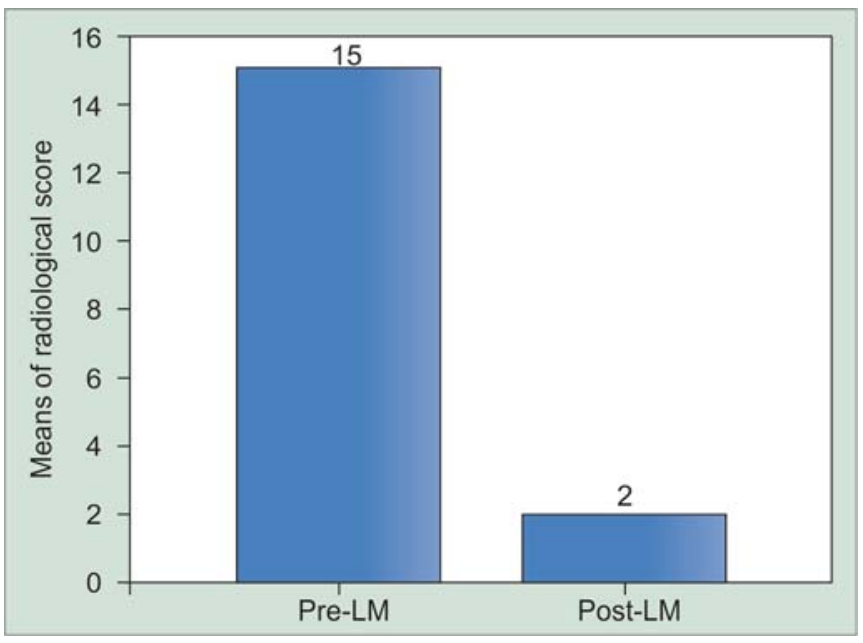

Fig. 3: The mean of radiological scores, pre- and 8-week postoperatively, using Lund-Mackay scoring system [Pre-LM: Preoperative radiological (Lund-Mackay) score; Post-LM: Postoperative radiological (Lund-Mackay) score] 


\begin{tabular}{|c|c|c|c|c|c|c|c|c|c|c|}
\hline \multicolumn{11}{|c|}{ Master Chart } \\
\hline Age & Sex & Pre-SS & Post-SS & Post-SS 6 & Pre-LM & Post-LM & Post-LM6 & Pre-ES & Post-ES & Post-ES6 \\
\hline 48 & $M$ & 2.1 & 1.3 & 1.4 & 24 & 7 & 8 & 10 & 4 & 5 \\
\hline 47 & $M$ & 2.5 & 0.91 & 1 & 24 & 1 & 3 & 12 & 0 & 2 \\
\hline 48 & $M$ & 1.67 & 0.33 & 0.4 & 10 & 4 & 5 & 4 & 5 & 3 \\
\hline 58 & $\mathrm{~F}$ & 2.5 & 0.91 & 1 & 21 & 4 & 23 & 12 & 3 & 2 \\
\hline 11 & $M$ & 1.4 & 0 & 1.6 & 17 & 0 & 2 & 8 & 1 & 2 \\
\hline 17 & $M$ & 1.58 & 0.33 & 0.44 & 12 & 2 & 3 & 6 & 1 & 1 \\
\hline 55 & $M$ & 1.91 & 0.33 & 0.44 & 8 & 2 & 3 & 4 & 2 & 1 \\
\hline 29 & $M$ & 2.5 & 0.42 & 0.52 & 24 & 4 & 5 & 12 & 1 & 4 \\
\hline 57 & $M$ & 2.8 & 1 & 1.2 & 23 & 10 & 8 & 11 & 1 & 3 \\
\hline 25 & $M$ & 2.1 & 0.42 & 0.52 & 10 & 0 & 2 & 5 & 5 & 4 \\
\hline 42 & $M$ & 2.42 & 0.33 & 0.33 & 18 & 2 & 3 & 8 & 1 & 3 \\
\hline 14 & $\mathrm{~F}$ & 2.5 & 0.25 & 0.33 & 17 & 3 & 4 & 9 & 1 & 3 \\
\hline 51 & $M$ & 1.91 & 0 & 1.96 & 13 & 0 & 2 & 5 & 2 & 3 \\
\hline 54 & $\mathrm{~F}$ & 2.1 & 0.42 & 0.52 & 14 & 4 & 6 & 8 & 3 & 2 \\
\hline 42 & $M$ & 2.2 & 0.17 & 0.22 & 14 & 0 & 2 & 8 & 1 & 3 \\
\hline 61 & $\mathrm{~F}$ & 1.84 & 0.25 & 0.33 & 9 & 2 & 4 & 5 & 3 & 4 \\
\hline 47 & $\mathrm{~F}$ & 2.5 & 0.17 & 0.33 & 11 & 1 & 4 & 5 & 1 & 2 \\
\hline 26 & $M$ & 2.1 & 0.33 & 0.42 & 11 & 2 & 4 & 4 & 3 & 4 \\
\hline 48 & $\mathrm{~F}$ & 1.67 & 0.25 & 0.33 & 6 & 1 & 4 & 4 & 3 & 4 \\
\hline 38 & $M$ & 1.75 & 0.33 & 0.52 & 15 & 0 & 2 & 8 & 1 & 2 \\
\hline
\end{tabular}

M: Male; F : Female; Pre-SS: Preoperative symptom score; Post-SS: Postoperative symptom score; Post-SS6: Postoperative symptom score after 6 months; Pre-LM: Preoperative radiological (Lund-Mackay) score; Post-LM: Postoperative radiological score; Post-LM6: Postoperative radiological score after 6 months; Pre-ES: Preoperative endoscopic score; Post-ES: Postoperative endoscopic score; Post-ES6: Postoperative endoscopic score after 6 months

\section{CORRELATIONS}

We found significant variance in the correlations between the three parameters, before and after surgery.

Preoperatively, all parameters correlated well statistically. The endoscopic and radiological scores correlated the most (Fig. 5). M oderate positive correlation was also noted between the symptom- endoscopic and radiological scores (Figs 4 and 6).

However, postoperatively these correlations started to change, mainly in decreasing fashion.

The symptoms- radiology correlation level-improved mildly at the 8-week stage (0.6-0.7) before reducing to 0.2 at 6 months. The symptoms- endoscopy correlationreduced from 0.6 to 0.14 and then to 0.025 at the second evaluation. The most significant loss of correlation was between the endoscopic and radiological scores ( 0.95 to 0.14 to negative value).

Table 3 shows all the correlations calculated in this study.

Thus, all our parameters of evaluation of CRS appeared to correlate well prior to surgery, while gradually losing their inter-relations with time after FESS. This was despite the statistical fact of maintained improvement of these parameters after the surgery.

\section{DISCUSSION}

As with any other disease, detailed history, accurate examination and relevant investigations are essential for the diagnosis of CRS with or without nasal polyps. Symptom

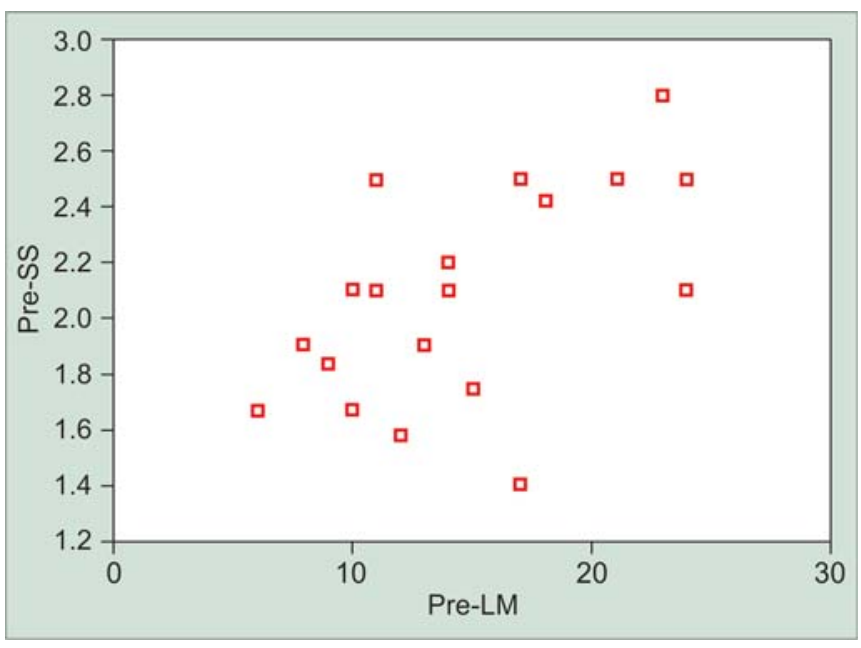

Fig. 4: The moderate correlation between preoperative symptom (SS) and radiological (LM) scores $(r=0.59)$

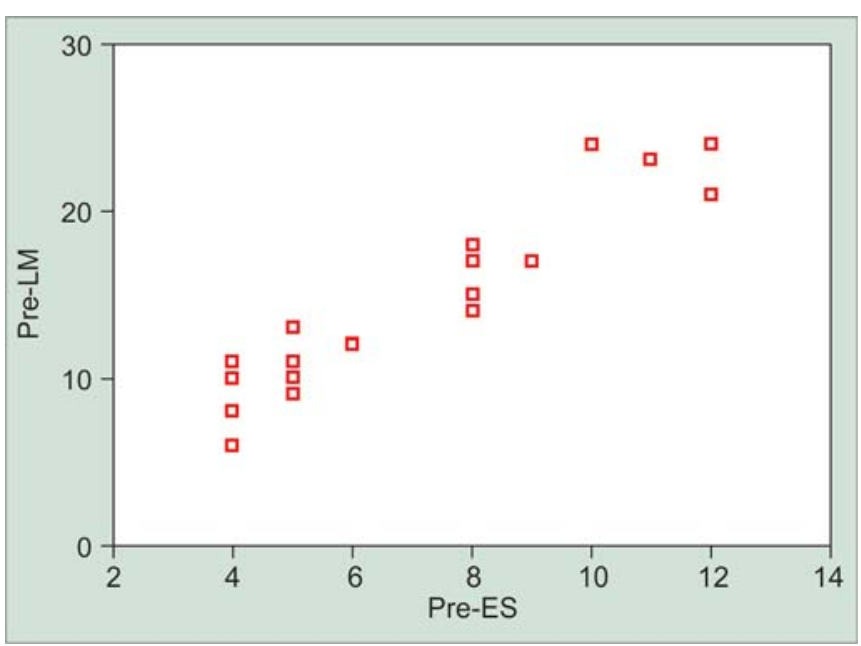

Fig. 5: The high correlation between preoperative radiological (LM) and endoscopic (ES) scores $(r=0.94)$ 
Correlations and Comparison between Repeat Computed Tomography Scores, Endoscopy Scores and Symptomatic

\begin{tabular}{|c|c|c|c|c|c|c|c|c|c|c|}
\hline & & Pre-sS & Post-SS & Post-SS6 & Pre-LM & Post-LM & Post-LM6 & Pre-ES & Post-ES & Post-ES6 \\
\hline Pre-SS & $\begin{array}{l}\text { Pearson correlation } \\
\text { sig. (2-tailed) } \\
\mathrm{N}\end{array}$ & $\begin{array}{l}1 \\
0.0 \\
20\end{array}$ & $\begin{array}{l}0.484^{*} \\
0.031 \\
20\end{array}$ & $\begin{array}{l}-0.050 \\
0.833 \\
20\end{array}$ & $\begin{array}{l}0.592^{* *} \\
0.006 \\
20\end{array}$ & $\begin{array}{l}0.462^{*} \\
0.040 \\
20\end{array}$ & $\begin{array}{l}0.373 \\
0.105 \\
20\end{array}$ & $\begin{array}{l}0.604^{* *} \\
0.005 \\
20\end{array}$ & $\begin{array}{l}-0.297 \\
0.203 \\
20\end{array}$ & $\begin{array}{l}0.124 \\
0.601 \\
20\end{array}$ \\
\hline Post-SS & $\begin{array}{l}\text { Pearson correlation } \\
\text { sig. (2-tailed) } \\
\mathrm{N}\end{array}$ & $\begin{array}{l}0.484^{*} \\
0.031 \\
20\end{array}$ & $\begin{array}{l}1 \\
0.0 \\
20\end{array}$ & $\begin{array}{l}0.263 \\
0.263 \\
20\end{array}$ & $\begin{array}{l}0.670^{* *} \\
0.001 \\
20\end{array}$ & $\begin{array}{l}0.719^{*} \\
0.000 \\
20\end{array}$ & $\begin{array}{l}0.572^{\text {** }} \\
0.008 \\
20\end{array}$ & $\begin{array}{l}0.628^{* *} \\
0.003 \\
20\end{array}$ & $\begin{array}{l}0.144 \\
0.546 \\
20\end{array}$ & $\begin{array}{l}0.210 \\
0.375 \\
20\end{array}$ \\
\hline Post-SS 6 & $\begin{array}{l}\text { Pearson correlation } \\
\text { sig. (2-tailed) } \\
\mathrm{N}\end{array}$ & $\begin{array}{l}-0.050 \\
0.833 \\
20 \\
\end{array}$ & $\begin{array}{l}0.263 \\
0.263 \\
20\end{array}$ & $\begin{array}{l}1 \\
0.0 \\
20\end{array}$ & $\begin{array}{l}0.453^{*} \\
0.045 \\
20\end{array}$ & $\begin{array}{l}0.178 \\
0.453 \\
20\end{array}$ & $\begin{array}{l}0.178 \\
0.453 \\
20\end{array}$ & $\begin{array}{l}0.310 \\
0.183 \\
20\end{array}$ & $\begin{array}{l}-0.052 \\
0.827 \\
20\end{array}$ & $\begin{array}{l}0.025 \\
0.915 \\
20\end{array}$ \\
\hline Pre-LM & $\begin{array}{l}\text { Pearson correlation } \\
\text { sig. (2-tailed) } \\
\mathrm{N}\end{array}$ & $\begin{array}{l}0.592^{* *} \\
0.006 \\
20\end{array}$ & $\begin{array}{l}0.670^{\text {** }} \\
0.001 \\
20\end{array}$ & $\begin{array}{l}0.453^{*} \\
0.045 \\
20\end{array}$ & $\begin{array}{l}1 \\
0.0 \\
20\end{array}$ & $\begin{array}{l}0.518^{*} \\
0.019 \\
20\end{array}$ & $\begin{array}{l}0.383 \\
0.096 \\
20\end{array}$ & $\begin{array}{l}0.946^{* *} \\
0.000 \\
20\end{array}$ & $\begin{array}{l}-0.382 \\
0.097 \\
20\end{array}$ & $\begin{array}{l}0.094 \\
0.693 \\
20\end{array}$ \\
\hline Post-LM & $\begin{array}{l}\text { Pearson correlation } \\
\text { sig. (2-tailed) } \\
\mathrm{N}\end{array}$ & $\begin{array}{l}0.462^{*} \\
0.040 \\
20 \\
\end{array}$ & $\begin{array}{l}0.719^{\text {** }} \\
0.000 \\
20\end{array}$ & $\begin{array}{l}0.178 \\
0.453 \\
20\end{array}$ & $\begin{array}{l}0.518 \\
0.019 \\
20\end{array}$ & $\begin{array}{l}1 \\
0.0 \\
20 \\
\end{array}$ & $\begin{array}{l}0.493^{*} \\
0.027 \\
20\end{array}$ & $\begin{array}{l}0.450^{*} \\
0.046 \\
20\end{array}$ & $\begin{array}{l}0.143 \\
0.547 \\
20\end{array}$ & $\begin{array}{l}0.233 \\
0.324 \\
20 \\
\end{array}$ \\
\hline Post-LM6 & $\begin{array}{l}\text { Pearson correlation } \\
\text { sig. ( } 2 \text {-tailed) } \\
\mathrm{N}\end{array}$ & $\begin{array}{l}0.373 \\
0.105 \\
20\end{array}$ & $\begin{array}{l}0.572^{\text {** }} \\
0.008 \\
20\end{array}$ & $\begin{array}{l}0.178 \\
0.453 \\
20\end{array}$ & $\begin{array}{l}0.383 \\
0.096 \\
20\end{array}$ & $\begin{array}{l}0.493^{*} \\
0.027 \\
20\end{array}$ & $\begin{array}{l}1 \\
0.0 \\
20\end{array}$ & $\begin{array}{l}0.472^{*} \\
0.036 \\
20\end{array}$ & $\begin{array}{l}0.230 \\
0.330 \\
20\end{array}$ & $\begin{array}{l}-0.026 \\
0.915 \\
20\end{array}$ \\
\hline Pre-ES & $\begin{array}{l}\text { Pearson correlation } \\
\text { sig. (2-tailed) } \\
\mathrm{N}\end{array}$ & $\begin{array}{l}0.604^{* *} \\
0.005 \\
20\end{array}$ & $\begin{array}{l}0.628^{\text {** }} \\
0.003 \\
20\end{array}$ & $\begin{array}{l}0.310 \\
0.183 \\
20\end{array}$ & $\begin{array}{l}0.946^{* *} \\
0.000 \\
20\end{array}$ & $\begin{array}{l}0.450^{*} \\
0.046 \\
20\end{array}$ & $\begin{array}{l}0.472^{*} \\
0.036 \\
20\end{array}$ & $\begin{array}{l}1 \\
0.0 \\
20\end{array}$ & $\begin{array}{l}-0.425 \\
0.062 \\
20\end{array}$ & $\begin{array}{l}-0.013 \\
955 \\
20\end{array}$ \\
\hline Post-ES & $\begin{array}{l}\text { Pearson correlation } \\
\text { sig. ( } 2 \text {-tailed) } \\
\mathrm{N}\end{array}$ & $\begin{array}{l}0.297 \\
0.203 \\
20\end{array}$ & $\begin{array}{l}0.144 \\
0.546 \\
20\end{array}$ & $\begin{array}{l}-0.052 \\
0.827 \\
20\end{array}$ & $\begin{array}{l}-0.382 \\
0.097 \\
20\end{array}$ & $\begin{array}{l}0.143 \\
0.547 \\
20\end{array}$ & $\begin{array}{l}0.230 \\
0.330 \\
20\end{array}$ & $\begin{array}{l}0.425 \\
0.062 \\
20\end{array}$ & $\begin{array}{l}1 \\
0.0 \\
20\end{array}$ & $\begin{array}{l}0.477^{*} \\
0.033 \\
20\end{array}$ \\
\hline Post-ES6 & $\begin{array}{l}\text { Pearson correlation } \\
\text { sig. (2-tailed) } \\
\mathrm{N}\end{array}$ & $\begin{array}{l}0.124 \\
0.601 \\
20\end{array}$ & $\begin{array}{l}0.210 \\
0.375 \\
20\end{array}$ & $\begin{array}{l}0.025 \\
0.915 \\
20\end{array}$ & $\begin{array}{l}0.094 \\
0.693 \\
20\end{array}$ & $\begin{array}{l}0.233 \\
0.324 \\
20\end{array}$ & $\begin{array}{l}-0.026 \\
0.915 \\
20\end{array}$ & $\begin{array}{l}-0.013 \\
0.955 \\
20\end{array}$ & $\begin{array}{l}0.477^{*} \\
0.033 \\
20\end{array}$ & $\begin{array}{l}1 \\
0.0 \\
20\end{array}$ \\
\hline
\end{tabular}

*Correlation is significant at the 0.05 level $\left(2\right.$-tailed); ${ }^{* *}$ Correlation is significant at the 0.01 level (2-tailed).

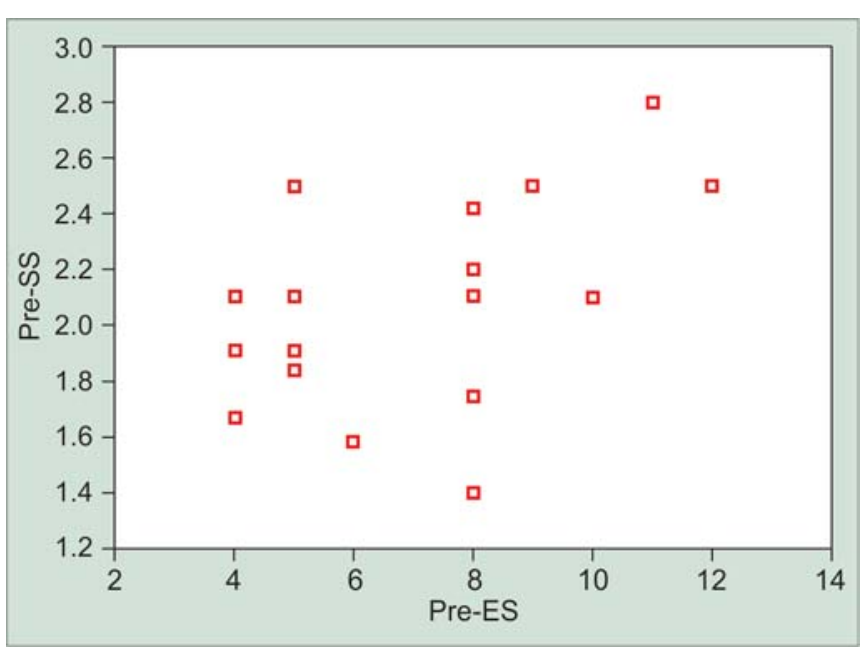

Fig. 6: Moderate degree of correlation between preoperative symptom (SS) and endoscopic (ES) scores $(r=0.61)$

questionnaires, diagnostic nasal endoscopy and CT PNS are the accepted modalities for the above. Treatment protocols are also largely well established; medical management and, where indicated, surgery in the form of endoscopic sinus clearance of disease by FESS.
The next step in scientific enquiry has been to quantify the various modalities and to achieve a surgical audit. To this end, researchers have described various methods in the literature, as outlined in the introduction. We have endeavored to study the correlations between the said three parameters of evaluation of CRS, before and after surgery. This is a pilot study in the Indian setting, and the first of its kind in its scope, involving repeat postoperative scans.

Sample size: A fter reviewing the rel evant literature, the sample size was estimated using a standard formula for comparing the mean values of variables, such as symptom score, radiological score and endoscopic score. ${ }^{15} \mathrm{~T}$ he sample size calculated was 70 with a confidence level of $95 \%$ and power of $80 \%$. Inadequate sample size was mainly due to increased financial requirement of the patient.

\section{Scoring Systems}

RSI has been reliably used to quantify the several symptoms experienced by the patient of CRS. ${ }^{10}$ W e have used a slightly modified version of the same, whereby the questions not directly related to the symptomatology of CRS have been excluded (e.g. use of prior medications and socioeconomic 
handicaps due to the symptoms). There was also concern regarding the issue of patient understanding and comfort level with too complex a questionnaire; hence certain simplifications were done.

The findings on diagnostic nasal endoscopy were meticulously noted and staged using the Lund-M ackay scoring system. $V$ arious studies have used different methods for this; not all of these named.

CT PNS is now the proven investigation of choice for the radiological exploration of CRS with or without NP. It provides objective evidence of the disease and its precise topography. It can also be used to assess response to surgery. ${ }^{16} 0$ bjectivity can be further enhanced if a grading of the disease findings on CT is done. To this end, the A merican A cademy of Otolaryngology Task Force on rhinosinusitis has recommended the L und-M ackay system for radiographic staging. ${ }^{17}$ Theoretically, the score thus calculated could be an indicator of the disease severity. However, many studies have failed to establish this correlation. Thus, many authors have advocated the use of CT scan merely as a tool in evaluating paranasal sinus anatomy. ${ }^{18}$

\section{Preoperative Correlations}

All the preoperative parameters showed good intercorrelations in our study. This is in contrast with other studies, especially with regard to symptom-CT correlation. ${ }^{14,19,20}$ Satish N air, in a large Indian study, found a strong association between symptom severity and a modified CT scoring system. ${ }^{21} \mathrm{H}$ ow ever, he states that they may not al ways correlate due to incidental CT findings and/ or lack of subjective issues. It has also been hypothesized that patients with worse disease, based on CT scan, experience greater symptomatic improvement after surgical treatment. Toros et al in one study proved that endoscopic sinus surgery provides significant symptomatic relief and endoscopically evident healing in patients with CRS and nasal polyps. ${ }^{22}$ They recorded the preoperative symptom and endoscopic scores, and postoperative symptom and endoscopic scores using VAS.

Our study has focussed on the correlations between all the three parameters.

Our results show a clear correlation between symptoms and Lund-M ackay scores preoperatively (Pearson correlation coefficient, $r=0.59, p=0.006$ ). This is possibly attributable to the precise timing of the scans viz immediately after a full course protocol treatment as outlined earlier. This effect has been documented in literature. ${ }^{15}$ One large study found no correlation between SNOT20 and CSS scores on the one hand and CT scores on the other. ${ }^{13} \mathrm{~A}$ nother smaller study found correlation between a high symptom score (rhinosinusitis task force, $0-10$ ) and CT findings. ${ }^{23}$ Thus, studies reporting poor correlation between symptoms and $\mathrm{CT}$ have tended to use differing symptom scoring systems. Could it be that our RSI-based questionnaire tends to associate better with the $C T$ findings? W e could perhaps hypothesize thus.

On the other hand, endoscopic scoring systems differ as a function of the presence of allergy, which results in abnormally high score. The endoscopy-CT scan correlation also has differing reports from studies; majority indicating a positive score. Stankiewicz et al reported poor correlation, while Casiano et al, using a flexible endoscope and Rosbe et al have reported fair to good correlation..$^{24-26}$ We could not ascertain the scoring system used in these studies. W e found a strong correlation between our endoscopy and $C T$ (both L und-M ackay systems) scores $(r=0.94, p<0.001)$. We presume that this could be due to the bias in terms of the chosen cases being definitively indicated for surgery. The endoscopy and symptom scores al so correlated well ( $r=$ $0.6, p<0.01$ ).

\section{Surgery and Postoperative Period}

FESS is now the accepted modality of surgical management of CRS with or without N $P$ in the setting of failure of medical management. In our center, we have been doing this surgery with almost all state-of-the-art equipment like the microdebrider, endoscopes of various angles and associated camera for demonstration and recording purpose. The 20 patients selected for the study underwent the standard steps of FESS involving clearance of the CT-involved sinuses and OM Cs. Postoperative care consisted of saline douches, and resumption of antiallergic medication (oral antihistaminic and nasal steroid spray) after 2 weeks. Oral steroid was not part of the routine protocol. Only these study patients underwent repeat CT scans at 8 weeks and 6 months, postoperatively; otherwise, this is not the norm. Review duration was first week, third week, eighth week and then monthly. The repeat scorings were done at 8 weeks and 6 months, as already mentioned.

\section{Comparisons}

Improvement after surgery has been along expected lines, as reported in various studies. ${ }^{14}$ However, all three parameters worsened slightly at the 6-month period, but the improvement, as compared to preoperative scores, continued to be statistically significant. Symptom scores reduced from a mean preoperative value of $2.103 \pm 0.383$ to $0.423 \pm 0.340$ $(p<0.001)$ and through to $0.69 \pm 0.50(p<0.001)$. Endoscopic scores changed from $7.40 \pm 2.89$ to $2.10 \pm 1.45$ 
Correlations and Comparison between Repeat Computed Tomography Scores, Endoscopy Scores and Symptomatic

$(p<0.001)$ first and $2.85 \pm 1.09(p<0.001)$ at the last review. CT scores showed improvement from a mean value of $15.05 \pm 5.73$ to $2.45 \pm 2.56$, and then to $4.85 \pm 4.64$ ( $p<$ $0.001)$. The variation in the mean and standard deviation is probably due to the difference in the preoperative and postoperative val ues. These statistics reinforce the need for continuance of medical management with regular reviews.

\section{Postoperative Correlations}

There have been very few studies reporting postoperative correlations. Logically, one would expect these to parallel the preoperative statistics, given the consistent improvement in all the parameters. William Ryan et al have, in a recent study, documented poor correlation between symptoms and both endoscopic and CT findings, postoperatively, but with endoscopy and CT findings correlating well. ${ }^{27}$

Can the severity of CRS on postoperative CT scans predict the severity of residual symptoms? Pierre B onfils et al in their study on 114 patients detected 11 asymptomatic mucoceles. ${ }^{15}$ They found a correlation between symptom and $\mathrm{CT}$ scores after surgery.

In our study, we have found mostly a negative trend, i.e. correlations have reduced toward the 6 -month period. Only the symptoms-CT association improved minimally at first ( $r=0.72-0.59$ ) before reducing to $r=0.18$. The symptoms-endoscopy correlation reduced from 0.6 to 0.14 and then to 0.025 at the second evaluation. In dramatic contrast was the loss of correlation between the endoscopic and radiological scores ( 0.95 to 0.14 to negative value). How is this explained? It could be that the L und-M ackay scoring system for endoscopic findings is not so valid in the postoperative scenario. A Iternately, creation of a separate CT scoring for postoperative findings should be considered, paralleling the endoscopic findings chart. Probably, the correlations would be re-established in case of genuine recurrence of disease (sinonasal polyps).

Also, it can be concluded that there is no definitive role for the repeat postoperative CT scan. Given the financial strain and radiation risk, a sustained review with the symptom questionnaire and endoscopic check should suffice.

\section{Limitations}

This study has suffered from the major limitation of inadequate sample size due to increased financial requirement for postoperative $\mathrm{CT}$ scan and limited study period compared to most studies in literature. There has also been failure to present the data on sinus opacification of individual sinus, pre- and postoperatively and to document the prevalence of anatomical abnormalities, asymptomatic mucoceles and osteitic changes in the CT images. W e have al so not assessed baseline symptom scores before starting the medical therapy.

\section{CONCLUSION}

It is of great clinical and academic use to have an objective record of the criteria of diagnosis of CRS with or without sinonasal polyposis. Our study has shown good to strong correlation between the symptoms, endoscopic findings and CT images. A n extensive database of these, using the RSIbased and Lund-M ackay scoring systems, could be built at a multi-institutional level to further explore the issue.

These figures are also useful as a preoperative index of those cases of CRS going in for surgical treatment (FESS). This study has shown sustained statistically significant improvement of all the scores, thus achieving a positive surgical audit.

In this pilot study of two repeat recording of postoperative scores, correlations between the parameters have been negative over a period after FESS. We feel that the symptomatic relief felt by the patient after FESS may not be reflected in the endoscopic and CT findings. Hence, the preoperative scoring systems for CRS may not apply to the postoperative case. It would be not of much clinical use to expose the patient to the extra radiation and financial constraint of repeat scans. As such, we recommend the use of only symptom scoring charts and, possibly, a revised endoscopic scoring for the post-FESS cases.

\section{REFERENCES}

1. Hopkins, et al. Surgery audit for nasal polyposis and CRS. L aryngoscope 2009;119:2459-65.

2. A nand VK. Epidemiology and economic impact of rhinosinusitis. Ann Otol Rhinol L aryngol Suppl 2004;193:3-5.

3. Devaiah AK. A dult chronic rhinosinusitis: Diagnosis and dilemmas. Otolaryngol Clin N A m 2004;37(2):243-52.

4. Benninger M S, Ferguson BJ, Hadley J A, Hamilos DL, J acobs $M, K$ ennedy DW, et al. A dult chronic rhinosinusitis: D efinitions, diagnosis, epidemiology, and pathophysiology. Otolaryngol Head N eck Surg 2003;129(3 Suppl):1-32.

5. W rightED, A grawal S. I mpact of perioperative systemic steroids on surgical outcomes in patients with chronic rhinosinusitis with polyposis: Evaluation with the novel perioperative sinus endoscopy (POSE) scoring system. L aryngoscope Suppl 2007;117:1-28.

6. Report of the Rhinosinusitis Task Force Committee M eeting. A lexandria, V irginia, A ugust 17, 1996. O tolaryngol H ead Neck Surg 1997;117(3 Pt 2):S1-68.

7. Gliklich RE, M etson R. Technique for outcomes research in chronic sinusitis. Laryngoscope 1995;105:387-90.

8. Piccirillo J, M erritt], Richards M. Psychometric and clinimetric validity of the 20 -item sinonasal outcome test. Otolaryngol Head Neck Surg 2002;126:41-47.

9. Bhattacharyya N. A comparison of symptom scores and radiographic staging systems in chronic rhinosinusitis. A m J Rhinol 2005;19:175-79. 
10. B hattacharyya N. Clinical and symptom criteria for the accurate diagnosis chronic rhinosinusitis. Laryngoscope 2006;116(7 Suppl 110)(Pt 2).

11. Basu S, G eorgalas C, K umar B N, D esai S. Correlation between symptoms and radiological findings in patients with chronic rhinosinusitis: An evaluation study using the sinonasal assessment questionnai re and Lund-M ackay grading system. Eur A rch Otorhinolaryngol 2005;262(9):751-54.

12. Liu Z, W ang $H, L u X, Y$ ou $X G, G$ ao Q, Cui Y. Correlation between rhinosinusitis symptoms and objective sinus examinations. Lin Chung Er Bi Y an Hou Tou Jing Wai Ke Za Zhi 2007;21:483-85.

13. Wabnitz DA, Nair S, Wormald PJ. Correlation between preoperative symptom scores, qual ity-of-life questionnaires, and staging with computed tomography in patients with chronic rhinosinusitis. A m J R hinol 2005;19:91-96.

14. Bradely DT, Kountakis SE. Correlation between computed tomography scores and symptomatic improvement after endoscopic sinus surgery. L aryngoscope 2005;115(3):466-69.

15. Bonfils $P$, Tavernier $L$, Rahman $H A, M$ imoun $M, M$ alinvaud $D$. Evaluation of combined medical and surgical treatment in nasal polyposis-III. Correlation between symptoms and CT scores before and after surgery for nasal polyposis. A cta OtoL aryngologica 2008;128(3):318-23.

16. Bhattacharyya N. Computed tomographic staging and the fate of the dependent sinuses in revision endoscopic sinus surgery. A rch O tol Head-N eck Surg 1999;125:994-99.

17. Lund VJ, Mackay IS. Staging in rhinosinusitis. R hinology 1993;31:183-84.

18. Tolsdorff B. V irtual reality: A new paranasal sinus surgery simulator. Laryngoscope 2010;120:420-27.

19. Bhattacharyya N. Radiographic stage fails to predict symptom outcomes after endoscopic sinus surgery for chronic rhinosinusitis. Laryngoscope 2006;116(1):18-22.

20. Holbrook EH, Brown CL, Lyden ER, Leopold DA. Lack of significant correlation between rhinosinusitis symptoms and specific regions of sinus computer tomography scans. A m J Rhinol 2005;19:382-87.
21. N air S. Correlation between symptoms and radiological findings in patients of chronic rhinosinusitis: A modified radiological typing system. Rhinology 2009;47:181-86.

22. Toros SZ, B olukbasi S, N aiboglu B, Er B, A kkayanak C, N oshari $\mathrm{H}$, et al. Comparative outcomes of endoscopic sinus surgery in patients with chronic sinusitis and nasal polyps. Eur Arch Otorhinolaryngol 2007;264(9):1003-08.

23. A rango $P, K$ ountakis $S E$. Significance of computed tomography pathology in chronic rhinosinusitis. Laryngoscope 2001;111: 1779-82.

24. Stankiewicz JA, Chow JM. A diagnostic dilemma for chronic rhinosinusitis: Definition accuracy and validity. A m J Rhinol 2002;16:199-202.

25. Casiano RR. Correlation of clinical examination with computer tomography in paranasal sinus disease. A m J Rhinol 1997;11: 193-96.

26. Rosbe K W, J ones K R. U seful ness of patient symptoms and nasal endoscopy in the diagnosis of chronic sinusitis. A m J Rhinol $1998 ; 12: 167-71$

27. Ryan WR, Ramachandra T, Hwang PH. Correlations between symptoms, nasal endoscopy, and in-office computed tomography in postsurgical chronic rhinosinusitis patients. Laryngoscope 2011;121:674-78.

\section{ABOUT THE AUTHORS}

\section{NV Deepthi}

Postgraduate Student, D epartment of ENT, A mrita Institute of M edical Sciences, K ochi, K erala, India

\section{Unnikrishnan K Menon (Corresponding Author)}

A ssistant Professor, D epartment of ENT, A mrita Institute of M edical Sciences, Kochi, Kerala-682041, India, Phone: 0091-9447831755 Fax: 0091-484 2802051, e-mail: unnikrishnanmenon8@gmail.com

\section{Indudharan R Menon}

Professor and H ead, D epartment of ENT, A mrita Institute of M edical Sciences, K ochi, K erala, India 